Revista Destaques Acadêmicos, Lajeado, v. 9, n. 3, 2017. ISSN 2176-3070 DOI: http://dx.doi.org/10.22410/issn.2176-3070.v9i3a2017.1508 www.univates.br/revistas

\title{
INCIDÊNCIA DE PARASITOS INTESTINAIS EM CRIANÇAS DAS ESCOLAS MUNICIPAIS DE EDUCAÇÃO INFANTIL E ENSINO FUNDAMENTAL DE UM MUNICÍPIO DO INTERIOR DO RIO GRANDE DO SUL
}

\author{
Makely Anny Fachini Delazeri ${ }^{1}$, Gabriela kniphoff da Silva Lawisch ${ }^{2}$
}

\begin{abstract}
Resumo: As enteroparasitoses ainda são consideradas um grave problema de saúde pública mundial, principalmente em países em desenvolvimento, em consequência dos altos índices de morbidade e mortalidade. A transmissão de parasitas intestinais está relacionada a insuficientes hábitos de higiene e saneamento básico, além de contato direto entre indivíduos contaminados, favorecido por locais fechados. A população infantil é mais suscetível às infecções parasitárias, uma vez que apresentam imunidade imatura e pouco conhecimento sobre os hábitos de higiene. Considerando a importância das infecções por parasitos intestinais na pediatria, o objetivo do trabalho consistiu em verificar a incidência de enteroparasitos intestinais em crianças das Escolas Municipais de Educação Infantil e Ensino Fundamental de um município do interior do Rio Grande do Sul. No referido estudo, foram analisadas 55 amostras fecais, submetidas ao método de Hoffman, Pons e Janer, em abril de 2017. Para cada criança realizou-se apenas uma coleta. Neste cenário, entre todas as amostras analisadas, apenas três (5,5\%) apresentaram positividade para Giardia lamblia. Conclui-se que a baixa incidência de parasitos intestinais está relacionada com adequadas práticas de higiene e tratamento da água, evidenciando boas situações econômica, social, cultural e ambiental do meio escolar, familiar e comunitário.
\end{abstract}

Palavras-chave: Parasitoses. População infantil. Instituto de ensino municipal. EPF.

\section{INTRODUÇÃO}

As parasitoses intestinais são detectadas frequentemente em seres humanos, em países em desenvolvimento, como o Brasil, sendo consideradas um importante problema de saúde pública (BASSO et al., 2008). Os parasitos intestinais podem ser identificados em áreas urbanas e agrícolas, possuindo

1 Graduanda em Biomedicina da Univates, Lajeado, RS, Brasil. Email: makely_anny@hotmail.com.

2 Biomédica, mestre e doutoranda em Genética e Biologia Molecular pela UFRGS, professora da Univates, Lajeado, RS, Brasil. Email:gkdsilva@univates.br 
ampla disseminação geográfica (FONSECA et al., 2010). No mundo, aproximadamente 2 bilhões de indivíduos apresentam infecção por helmintos, destes, cerca de 300 milhões manifestam morbidade importante, no qual as crianças em idade escolar são as mais acometidas com a doença (WHO, 2016; OPS/WHO, 2014).

A prevalência das doenças parasitárias intestinais está relacionada com a combinação de fatores, como água e alimentos contaminados, precárias instalações sanitárias; práticas de higiene desapropriadas; faixa etária e escolaridade do hospedeiro (MACHADO et al., 1999). O predomínio da contaminação por esses parasitos obteve pequeno declínio em todos os níveis sociais (FERREIRA; FERREIRA; MONTEIRO, 2000), porém, ainda apresentase como bom indicador das condições econômicas, sociais e culturais de uma população (KOMAGOME et al., 2007).

Os humanos, normalmente, apresentam infecções por parasitos entéricos, com relevante contágio por helmintoses, como: Ascaridíase, Tricuríase, Enterobíase e Ancilostomíase. Já nas protozooses intestinais, destacam-se a Giardíase e a Amebíase (TOSCANI et al., 2007). O predomínio da contaminação se dá na infância, isto porque a classe infantil tem como elemento significativo para a disseminação de parasitos intestinais o baixo conhecimento sobre hábitos de higiene e a integração em aglomerações nas instituições de ensino e sistema imunológico imaturo (ZAIDEN et al., 2008).

As infecções por parasitos intestinais podem causar anemia; má absorção de nutrientes; diarreia; náuseas; vômitos; perda de peso; úlceras intestinais; inflamação do peritônio; obstrução entérica; irritação e transtorno de sono (KUCIK, MARTIN, SORTOR; 2004), além de comprometer o desempenho intelectual dos seus portadores. Todas essas patologias podem trazer consequências mais graves ao ocorrerem em crianças, comprometendo o desenvolvimento físico, cognitivo e social (FERREIRA et al., 2002). Devido a isto, a pesquisa teve como objetivo avaliar a incidência de parasitos intestinais em crianças das Escolas Municipais de Educação Infantil e Ensino Fundamental de um município do Interior do Rio Grande do Sul.

\section{MATERIAIS E MÉTODOS}

Durante o mês de abril de 2017 foram coletadas 55 amostras fecais de alunos de Escolas Municipais de Educação Infantil e Ensino Fundamental, de ambos os sexos, provenientes de um município do Interior do Rio Grande do Sul. Foi realizada uma única coleta de cada indivíduo para Exame Parasitológico de Fezes (EPF).

As amostras foram coletadas pelos pais e/ou responsáveis, em frascos estéreis adequados para coleta de fezes, devidamente rotulados, com instruções de coleta pregressas. Juntamente às coletas, um questionário com perguntas abordando questões de hábitos de vida e hábitos alimentares das crianças 
foram respondidos pelos responsáveis. As amostras foram acondicionadas em refrigeração até serem transportadas para o laboratório de Análises Clínicas Univates, onde foram analisadas.

Todas as crianças com idade entre quatro e oito anos e com a apresentação da Autorização a partir do Termo de Consentimento Livre e Esclarecido (TCLE) e do Termo de Assentimento foram incluídas na pesquisa.

As análises foram realizadas a partir do método de Hoffman, Pons e Janer ou Lutz. Técnica que consiste, por sedimentação espontânea, concentrar as formas parasitárias para identificação de ovos, cistos e larvas de parasitos entéricos intestinais (CARLI, 1994).

Um laudo foi entregue aos pais e/ou responsáveis dos alunos, perante orientação da pesquisadora conforme resultados da pesquisa, sendo possível, por parte dos pais, tomar as medidas cabíveis para a situação da criança.

Os resultados obtidos na pesquisa foram tabulados em planilha no Microsoft ${ }^{\circledR}$ Office ${ }^{\circledR}$ Excel, para avaliação das frequências absolutas e relativas.

O presente estudo foi aprovado pelo Comitê de Ética em Pesquisa da Univates (Coep/Univates) sob o número do protocolo 1.976.455.

\section{RESULTADOS}

Dos 107 alunos que integram as Escolas Municipais de Educação Infantil e Ensino Fundamental, $55(51,40 \%)$ participaram da pesquisa, e destes, 30 (54,5\%) são do sexo masculino e $25(45,5 \%)$ do sexo feminino, com idades entre quatro e oito anos, sendo que $32(58,2 \%)$ moram na zona rural do município. De todos os participantes, 28 (50,9\%) comunicaram ter tomado medicamentos antiparasitários no último ano; $27(49,1 \%)$ nunca realizaram exame parasitológico de fezes (EPF) e $46(83,6 \%)$ tinham contato com animais como, cachorro, gato e aves. Além disso, $54(98,2 \%)$ informaram que costumam lavar as mãos antes das refeições. Os demais dados obtidos a partir dos questionários estão apresentados na Tabela 1 . Das 55 crianças avaliadas, apenas três $(5,5 \%)$ apresentaram positividade para parasitose, sendo identificado o parasita Giardia lamblia. 
Tabela 1 - Dados obtidos por meio do questionário aos pais e/ou responsáveis

\begin{tabular}{l|c}
\hline \multicolumn{1}{c|}{ Questões } & $\begin{array}{c}\text { Escolas de Educação Infantil e } \\
\text { Ensino Fundamental }\end{array}$ \\
\hline 1. $\begin{array}{l}\text { Abastecimento de água } \\
\text { CORSAN (Distribuidora de água) } \\
\text { Poço artesiano }\end{array}$ & $24(43,6 \%)$ \\
Poço e Fonte & $22(40,0 \%)$ \\
Fonte & $4(7,3 \%)$ \\
2. Origem dos alimentos & $5(9,1 \%)$ \\
Mercado & \\
Mercado e horta própria & $8(14,5 \%)$ \\
Horta própria & $20(36,4 \%)$ \\
3. Lavagem dos alimentos & $27(49,1 \%)$ \\
Água & $49(89,1 \%)$ \\
Água e cloro & $5(9,1 \%)$ \\
Água e hipoclorito & $1(1,8 \%)$ \\
4. Hábito de brincar com pés descalços & \\
Sim & $43(78,2 \%)$ \\
Não & $12(21,8 \%)$ \\
5. Hábito de brincar na areia ou terra & $49(89,1 \%)$ \\
Sim & $6(10,9 \%)$ \\
Não & \\
\hline
\end{tabular}

Fonte: Do autor.

Das três crianças que apresentaram positividade para enteroparasitas intestinais, duas $(66,67 \%)$ residem na zona urbana do município, duas $(66,67 \%)$ fizeram uso de medicamento antiparasitário no último ano, duas $(66,67 \%)$ nunca realizaram exame parasitológico de fezes, duas (66,67\%) tinham contato com animais (como cachorro e gato e aves) e as três (100\%) lavavam as mãos antes das refeições. Além disso, uma $(33,33 \%)$ possui abastecimento de água a partir da distribuidora local, uma $(33,33 \%)$ por fonte e outra $(33,33 \%)$ por poço artesiano. Somente uma $(33,33 \%)$ criança tinha alimentos como verdura, legumes e frutas originados de horta própria, duas $(66,67 \%)$ possuem hábito de brincar com pés descalços e hábito de brincar na areia ou terra.

\section{DISCUSSÃO}

Segundo Basso et al. (2008) as doenças enteroparasitárias são indicadores do desenvolvimento social, econômico e ambiental de uma população, além de ser um grave problema de saúde pública, uma vez que a contaminação está relacionada com os hábitos de higiene, instalações sanitárias, faixa etária 
e grau de escolaridade. Os indivíduos que apresentam maior vulnerabilidade aos agravos da infecção parasitária são as crianças, devido principalmente ao frágil sistema imunológico e necessidade de atenção alheia (MELO; FERRAZ; ALEIXO, 2010). Como consequência, as infecções por parasitos intestinais podem levar a quadros de diarreia, anemia e desnutrição afetando o progresso físico e intelectual das crianças, principalmente em idade escolar (ABREU et al., 2014).

A incidência de parasitos intestinais nas crianças do estudo foi abaixo da média $(5,5 \%)$, quando comparadas a trabalhos anteriores como, por exemplo, em escolares de dois a quinze anos de idade da rede pública de Mirassol (SP) com positividade de 30,3\% (BELLOTO et al., 2011). Outro estudo realizado com escolares de seis a quatorze anos de Caxias do Sul (RS) demonstrou $58 \%$ de amostras positivas (BASSO et al., 2008). Ainda, em crianças de três a seis anos de Belo Horizonte (MG), a presença de parasitos intestinais foi de $24,6 \%$ das amostras analisadas (MENEZES et al., 2008). Por outro lado, os dados obtidos no presente estudo estão de acordo com os dados publicados por Gross e Silva (2016), obtidos em uma escola infantil pública e uma escola infantil comunitária, com crianças recém-nascidas até cinco anos de idade, apresentando uma positividade para enteroparasitos intestinais de $8 \%$ na escola pública e $6,25 \%$ na escola comunitária. Possivelmente, os resultados se assemelham devido a ambos os estudos serem oriundos de cidades de pequeno porte localizadas no interior do Rio Grande do Sul.

O protozoário Giardia lamblia foi o único parasito identificado, sendo este o agente etiológico da Giardíase, normalmente diagnosticada em crianças com idade inferior a 10 anos, independente da renda familiar (CIMERMAN; CIMERMAN, 1999). A transmissão pode ocorrer através da ingestão de cistos maduros em água sem tratamento adequado, alimentos contaminados por insetos e partículas de dejetos fecais em suspensão no ar ou de indivíduo para indivíduo em locais com aglomeração de pessoas (SANTANA et al., 2014). A infecção pode permanecer assintomática, ou apresentar sintomas como diarreia, dor abdominal, gases, vômito, perda de peso, má absorção de nutrientes e gorduras como, vitaminas A, D, E, K, vitamina B12, ferro, xilose e lactose e consequentemente a desnutrição (NETO et al., 2008; REY, 2001; WRIGHT; TOMKINS; RIDLEY, 1977).

Os resultados do presente estudo mostram que a frequência do protozoário Giardia lamblia ainda é ocorrente em escolares. Em uma pesquisa realizada na região de Uberlândia (MG), em crianças de seis meses a seis anos de idade, foi observada uma positividade de $29,3 \%$ das amostras, sendo a Giardia lamblia $(19,2 \%)$ o parasita intestinal de maior prevalência (GONÇALVES et al., 2011). No estudo de Belloto e colaboradores (2011), Mirrasol (SP), dos 30,3\% dos escolares que apresentaram positividade para algum parasito intestinal, a prevalência foi de Giardia lamblia, com 15,16\%. Porém, outros estudos apontam outros patógenos mais prevalentes na população infantil, como de Menezes 
et al. (2008), em Belo Horizonte (MG), no qual o parasita mais frequente em amostras fecais positivas foi o protozoário Entamoeba coli, considerado parasita comensal. Já em Caxias do Sul (RS), das amostras positivas, foi identificado com maior frequência o helminto Ascaris lumbricoides (BASSO et al., 2008), agente etiológico da Ascaridíase, que acarreta sintomas como, dor abdominal, diarreia, náusea, entre outros (DE CASTRO; BEYRODT, 2003; NETO et al., 2008).

$\mathrm{O}$ índice de parasitos observados no presente estudo foi baixo, o que aponta entre outros fatores, a boa qualidade da água consumida pelos munícipes (NETO et al., 2013), visto que essa é a principal via de contaminação de parasitos intestinais. Com base no questionário aplicado neste estudo, outros fatores como a lavagem das mãos antes das refeições e lavagem dos alimentos de forma correta (com água tratada adequadamente), diminuem o risco de contaminação por parasitos intestinais, mesmo quando na maioria dos casos a lavagem é feita somente com água (BLOOMFIELD, 2001; LUBY, 2001). Dessa forma, foi possível verificar que, quando são mantidas as principais medidas de profilaxia para enteroparasitoses intestinais, incluindo os hábitos de higiene e utilização de água tratada, o risco de contaminação por parasitos é baixo (DOREA et al., 1996), mesmo que as crianças tenham contato com o solo e animais em suas atividades diárias (conforme dados do questionário) (ARAUJO FILHO et al., 2011).

Além das boas condições de saneamento básico e higiene no ambiente familiar, a excelente estrutura escolar, que proporciona um ambiente de organização, higiene e limpeza tanto da escola, quanto das crianças e funcionários, é elemento essencial para a baixa incidência de parasitos intestinais encontrada no presente estudo. A análise do questionário dos três indivíduos contaminados demonstrou que a contaminação provavelmente ocorreu ao acaso, visto que não há nenhuma característica em comum entre os mesmos.

Entre as limitações do estudo, vale ressaltar que foi analisada apenas uma amostra fecal de cada educando, o que pode diminuir a possibilidade de se encontrar ovos, cistos ou larvas de parasitos intestinais, devido aos diferentes ciclos de vida dos mesmos. Ainda, foi utilizada apenas uma técnica de concentração de parasitos, baseada na sedimentação dos mesmos, o que favorece a identificação de ovos e cistos mais pesados. Dessa forma, não foi empregado o método de Rugai, Mattos e Brisola, específico para larvas de nematoides, o que pode ser a explicação para os resultados negativos para larvas de Strongyloides stercolaris. Outra técnica não aplicada foi a de Graham ou swab anal, para investigação de ovos Enterobius vermicularis. Também é importante destacar que mais da metade dos participantes fizeram uso de algum tipo de antiparasitário no último ano, o que provavelmente interferiu na baixa positividade parasitária encontrada, assim como no estudo de Frei, Juncansen e Ribeiro-Paes (2008). 
Além de receberem o resultado do Exame Parasitológico de Fezes, os pais e/ou responsáveis pelas crianças que obtiveram laudos positivos foram alertados sobre os meios de transmissão dos enteropasitos intestinais e orientados a procurar o médico da criança ou um posto de saúde.

\section{CONCLUSÃO}

Nas amostras analisadas houve uma baixa incidência de parasitos intestinais. Esses resultados indicam as boas condições de higiene das crianças, a boa qualidade da água e a correta administração dos alimentos ofertados para os escolares, evidenciando uma boa estrutura nos âmbitos familiar, escolar e comunitário. Porém, o estudo não reflete a realidade do Brasil, e sim de uma cidade de pequeno porte do interior do Rio Grande do Sul, visto que o país conta com ampla diversidade socioeconômica, ecológica e cultural.

\section{REFERÊNCIAS}

ABREU, L. K. et al. Prevalência e aspectos sócio-epidemiológicos de enteroparasitoses em crianças do centro municipal de educação infantil em Janiópolis - PR. SaBios: Rev. Saúde e Biol., Campo Maurão, v. 9, n. 3, p. 76 -84, Out./Dez. 2014. Disponível em: <http://revista.grupointegrado.br/revista/index.php/sabios2/article/view/1235>. Acesso em: 27 Abr. 2017.

ARAUJO FILHO, H. B. et al. Parasitoses intestinais se associam a menores índices de peso e estatura em escolares de baixo estrato socioeconômico. Rev. paul. pediatr., São Paulo, v. 29, n. 4, p. 521-528, Dec. 2011. Disponível em: <http:/ /www.scielo.br/scielo. php?script=sci_arttext\&pid=S0103-05822011000400009\&lng=en\&nrm=iso $>$. Acesso em: 05 May 2017.

BASSO, R. M. C. et al. Evolução da prevalência de parasitoses intestinais em escolares em Caxias do Sul, RS. Rev. Soc. Bras. Med. Trop., Uberaba, v. 41, n. 3, p. 263-268, June. 2008. Disponível em: <http:/ /www.scielo.br/scielo.php?script=sci arttext\&pid=S0037-86822008000300008\&lng=en\&nrm=iso $>$. Acesso em: 27 Abr. 2017.

BELLOTO, M. V. T. et al. Enteroparasitoses numa população de escolares da rede pública de ensino do Município de Mirassol, São Paulo, Brasil. Rev Pan-Amaz Saude, Ananindeua , v. 2, n. 1, p. 37-44, mar. 2011.Disponível em: <http:/ /scielo.iec.pa.gov. $\mathrm{br} /$ scielo.php?script=sci_arttext\&pid=S2176-62232011000100004\&lng=pt\&nrm=iso $>$. Acesso em: 28 Abr. 2017.

BLOOMFIELD, S.F. Preventing Infectious diseases in the domestic setting: a riskbased approach. Am. J. Infection Control, v.29, p.207-12, Aug. 2001. Disponível em: < http://www.ajicjournal.org/article/S0196-6553(01)04416-9/pdf>. Acesso em: 05 May 2017.

CARLI, G. A. de. Diagnostico laboratorial das parasitoses humanas: métodos e técnicas. Rio de Janeiro: MEDSI, 1994. 
CIMERMAN, B.; CIMERMAN, S. Parasitologia humana e seus fundamentos gerais. São Paulo: Atheneu, 1999.

DE CASTRO, C. G.; BEYRODT, C. G. P. Ações de enfermagem na prevenção de parasitoses intestinais em creches. Revista de Enfermagem UNISA, [S.1.], v. 4, p. 7680, 2003. Disponível em:< http://docplayer.com.br/12486781-Acoes-de-enfermagemna-prevencao-de-parasitoses-intestinais-em-creches.html>. Acesso em: 03 May. 2017.

DOREA, R. C. C. et al. Control of parasitic infections among school children in the peri-urban area of Botucatu, São Paulo, Brazil. Rev. Soc. Bras. Med. Trop., Uberaba , v. 29, n. 5, p. 425-430, Oct. 1996. Disponível em: <http:/ / www.scielo.br/scielo. php?script=sci_arttext\&pid=S0037-86821996000500004\&lng=en\&nrm=iso $>$. Acesso em: 05 May. 2017.

FERREIRA, H. S. et al. Saúde de populações marginalizadas: desnutrição, anemia e enteroparasitoses em crianças de uma favela do "Movimento dos Sem Teto", Maceió, Alagoas. Rev. Bras. Saude Mater. Infant., Recife, v. 2, n. 2, p. 177-185, Aug. 2002. Disponível em: <http:/ / www.scielo.br/scielo.php?script=sci_arttext\&pid=S151938292002000200010\&lng=en\&nrm=iso >. Acesso em: 27 Apr. 2017.

FERREIRA, M. U.; FERREIRA, C. S.; MONTEIRO, C. A. Tendência secular das parasitoses intestinais na infância na cidade de São Paulo (1984-1996). Rev. Saúde Pública, São Paulo, v. 34, n. 6, supl. p. 73-82, Dec. 2000. Disponível em: <http:/ /www. scielo.br/scielo.php?script=sci_arttext\&pid=S0034-89102000000700010\&lng=en\&nrm= iso>. Acesso em: 27 Abr. 2017.

FONSECA, E. O. L. et al. Prevalência e fatores associados às geo-helmintíases em crianças residentes em municípios com baixo IDH no Norte e Nordeste brasileiros.

Cad. Saúde Pública, Rio de Janeiro, v. 26, n. 1, p. 143-152, Jan. 2010. Disponível em: <http://www.scielo.br/scielo.php?script=sci_arttext\&pid=S0102-311X2010000100015 \&lng=en\&nrm=iso>. Acesso em: 27 Abr. 2017.

FREI, F.; JUNCANSEN, C.; RIBEIRO-PAES, J. T. Levantamento epidemiológico das parasitoses intestinais: viés analítico decorrente do tratamento profilático. Cad. Saúde Pública, Rio de Janeiro, v. 24, n. 12, p. 2919-2925, Dec. 2008. Disponível em:<http:/ / www.scielo.br/scielo.php?script=sci_arttext\&pid=S0102-311X2008001200021\&lng=en \&nrm=iso>. Acesso em:05 May. 2017.

GONCALVES, A. L. R. et al. Prevalência de parasitoses intestinais em crianças institucionalizadas na região de Uberlândia, Estado de Minas Gerais. Rev. Soc. Bras. Med. Trop., Uberaba, v. 44, n. 2, p. 191-193, Apr. 2011. Disponível em: <http:/ /www. scielo.br/scielo.php?script=sci_arttext\&pid=S0037-86822011000200013\&lng=en\&nrm= iso>. Acesso em: 02 Mai. 2017.

GROSS, A. A; SILVA, G.K. Incidência de enteroparasitos intestinais em uma escola infantil pública e uma escola intanfil comunitária, em um município do interior do Rio Grande do Sul. Destaques Acadêmicos, Lajeado, v. 8, n. 3, p. 50-57, 2016. Disponível em:< http:/ / univates.br/revistas/index.php/destaques/article/view/1053/1028>. Acesso em: 03 Mai. 2017. 
KOMAGOME, S. H. et al. Fatores de risco para infecção parasitária intestinal em crianças e funcionário de creches. Cienc. Cuid. Saúde, Maringá, v. 6, supl. 2, p. 442- 447, Mar. 2007. Disponível em: <http:/ / periodicos.uem.br/ojs/index.php/ CiencCuidSaude/article/view/5354> . Acesso em: 27 Abr. 2017.

KUCIK, C. J.; MARTIN, G. L; SORTOR, B. V. Common intestinal parasites. Am. Fam. Phys., Leawood, v. 69, n. 5, p.1161-1168, Mar. 2004. Disponível em: < http:/ /www. aafp.org/afp/2004/0301/p1161.html>. Acesso em: 27 Abr. 2017.

LUBY, S. The role of handwashing in improving hygiene and health in low-income countries. Am. J. Infection Control, v.29, p.239-40, Aug. 2001. Disponível em: < http:/ / www.ajicjournal.org/article/S0196-6553(01)67584-9/fulltext>. Acesso em: 05 May 2017.

MACHADO, R. C. et al. Giardíase e helmintíases em crianças de creches e escolas de $1^{\circ}$ e $2^{\circ}$ graus (públicas e privadas) da cidade de Mirassol (SP, Brasil). Rev. Soc. Bras. Med. Trop., Uberaba, v. 32, n. 6, p. 697-704, Dec. 1999. Disponível em: <http:/ /www. scielo.br/scielo.php?script=sci_arttext\&pid=S0037-86821999000600013\&lng=en\&nrm= iso>. Acesso em: 27 Abr. 2017.

MELO, E. M.; FERRAZ, F. N.; ALEIXO, D. L. Importância do estudo da prevalência de parasitos intestinais de crianças em idade escolar. SaBios: Rev. Saúde e Biol., Campo Maurão, v. 5, n. 1, p. 43-47, Jan./Jul. 2010. Disponível em: < http:/ / revista. grupointegrado.br/revista/index.php/sabios2/article/view/546>. Acesso em: 27 Abr. 2017.

MENEZES, A.L. et al. Prevalence of intestinal parasites in children from public daycare centers in the city of Belo Horizonte, Minas Gerais, Brazil. Rev. Inst. Med. trop. S. Paulo, São Paulo, v. 50, n. 1, p. 57-59, Feb. 2008 Disponível em: <http:/ /www. scielo.br/scielo.php?script=sci_arttext\&pid=S0036-46652008000100013\&lng=en\&nrm= iso>. Acesso em: 28 Abr. 2017.

NETO, A. P. S. et al. Ação e prevenção: uma avaliação parasitológica em manipuladores de alimentos e escolares. Revista Extendere, [S.1.], v. 2, n. 1, p. 23-40, jul./ dez. 2013. Disponível em: <http:/ / periodicos.uern.br/index.php/extendere/ article/view/776>. Acesso em: 03 Mai. 2017.

NETO, V. A. et al. Parasitologia: uma abordagem clínica. Rio de Janeiro: Elsevier, 2008.

OMS. Helmintiasis transmitidas por el suelo. 2016. Disponível em: <http:/ /www. who.int/mediacentre/factsheets/fs366/es/>. Acesso em: 27 Abr. 2017.

OPS/WHO. Helmintiasis transmitidas por el suelo. 2014. Disponível em: < http:/ / www2.paho.org/hq/index.php?option=com content\&view=article\&id=5747\&Itemid=4138 >. Acesso em: 27 Abr. 2017.

REY, L. Parasitologia: parasitos e doenças parasitárias do homem nas Américas e na África. 3. ed. Rio de Janeiro: Guanabara Koogan, 2001. 
SANTANA, L. A. et al. Atualidades sobre giardíase. Jornal Brasileiro de Medicina, [S.1.], v. 102, n. 1, Jan./Fev., 2014. Disponível em: <https:/ /www.researchgate.net/ publication/274074799_Atualidades_sobre_giardiase> . Acesso em: 28 Abr. 2017.

TOSCANI, N. V. et al. Desenvolvimento e análise de jogo educativo para crianças visando à prevenção de doenças parasitológicas. Interface (Botucatu), Botucatu , v. 11, n. 22, p. 281-294, Aug. 2007. Disponível em: <http:/ / www.scielo.br/scielo. php?script=sci_arttext\&pid=S1414-32832007000200008\&lng=en\&nrm=iso $>$. Acesso em: 27 Abr. 2017.

World Health Organization. The global burden of disease: 2004 update. Geneva: WHO, 2008. Disponível em: <http:/ / www.who.int/healthinfo/global_burden_ disease/2004_report_update/en/. Acesso em: 28 Abr. 2017.

WRIGHT, S. G.; TOMKINS A. M.; RIDLEY, D. S. Giardiasis: clinical and therapeutic aspects. Gut., London, v. 18, n. 5, p. 343-350, May. 1977. Disponível em: <https:/ / www.ncbi.nlm.nih.gov/pubmed/873319>. Acesso em: 28 Abr. 2017.

ZAIDEN, M. F. et al. Epidemiologia das parasitoses intestinais em crianças de creches de Rio Verde - GO. Medicina, Ribeirão Preto, v. 41, n. 2, p. 182-187, Abr./Jun., 2008. Disponível em:<http:/ /www.revistas.usp.br/rmrp/article/view/265/266>. Acesso em: 27 Abr. 2017. 